\title{
A Comprehensive Survey of Sensor Deployment Strategies to Diagnose Discrete-Part Manufacturing System
}

\author{
Kang $\mathrm{He}^{1,2}$, Minping $\mathrm{Jia}^{2}$, Zhuanzhe Zhao ${ }^{3}$ and Nan Wang ${ }^{1, *}$ \\ ${ }^{1}$ Mine Machinery and Electronic Eng. Research Center, Suzhou University, Suzhou, China; \\ ${ }^{2}$ School of Mechanical Engineering, Southeast University, Nanjing, China; \\ ${ }^{3}$ Sch. of Mechanical and Automotive Eng., Anhui Polytechnic University, Wuhu, China. \\ *987408776@qq.com
}

Keywords: discrete-part manufacturing, key modules, sensor, technical complexity.

\begin{abstract}
This paper presents a broad overview of the various sensor placement strategies to diagnose discrete-part manufacturing system. Due to the technical complexity, the performance of sensor system would be cursed by any modules of sensor placement strategies. Therefore, the current state of the sensor placement strategies is outlined with three key modules, namely cause-effect relationship model, optimization basis, and optimization algorithm, are surveyed in detail. The challenges faced by industry and academia are discussed and several principle conclusions are drawn, which could create a clear platform for the neophyte researchers for sensor deployment to diagnose discrete-part manufacturing system.
\end{abstract}

\section{Introduction}

To ensure the safety and reliability of manufacturing systems, Fault diagnosis is the critical and fundamental elements to diagnose the underlying fault reasons efficiently upon their occurrence [1-3]. Fault diagnosis is the action to identify if a system is deviating from the benchmark, and determine the potential root causes for this abnormal behaviours[4, 5]. A typical fault diagnosis action consists of three steps. Firstly, Key components, which are critical to system's reliability, safety and repair cost, etc., are identified based on the actual maintenance records; Secondly, sensors are selected and located to pick up signal signatures to faults, and finally, transitional data read by sensors is processed to identify the fault root causes. Therefore, fault diagnosis is widely used in manufacturing industry, such as Tool condition monitoring(TCM) [6,7], rotary machines [8, 9], assembly processes[10, 11], and stamping process[12], etc. Sensors and sensing technologies constitute the fundamental basis for fault diagnosis.

Regarded by MIT's technology review as one of the top ten emerging technologies that will change the world[13], the new technology of the distributed sensor network has given the researchers great excitement and serious attention. For complex manufacturing systems, duo to the difference on process configuration, sensor system homogeneity and variation sources[14], sensor deployment strategy also varies. Fig. 1 shows the complex chemical process and discrete-part manufacturing processes, respectively. Obviously, their process configuration is different. Compared with the mechanical manufacturing process, the operation data of most chemical processes not only has nonlinear relations, but also has multi-scale characteristics. When multiple sensors are considered, a homogeneous or heterogeneous sensor system is involved. In a general sense, the diagnosis-oriented sensor distribution strategy is concerned with the statistical properties of process variables[14]. Based on the abovementioned issues, considerable research has gone into the development of such sensor deployment strategy [15]. 

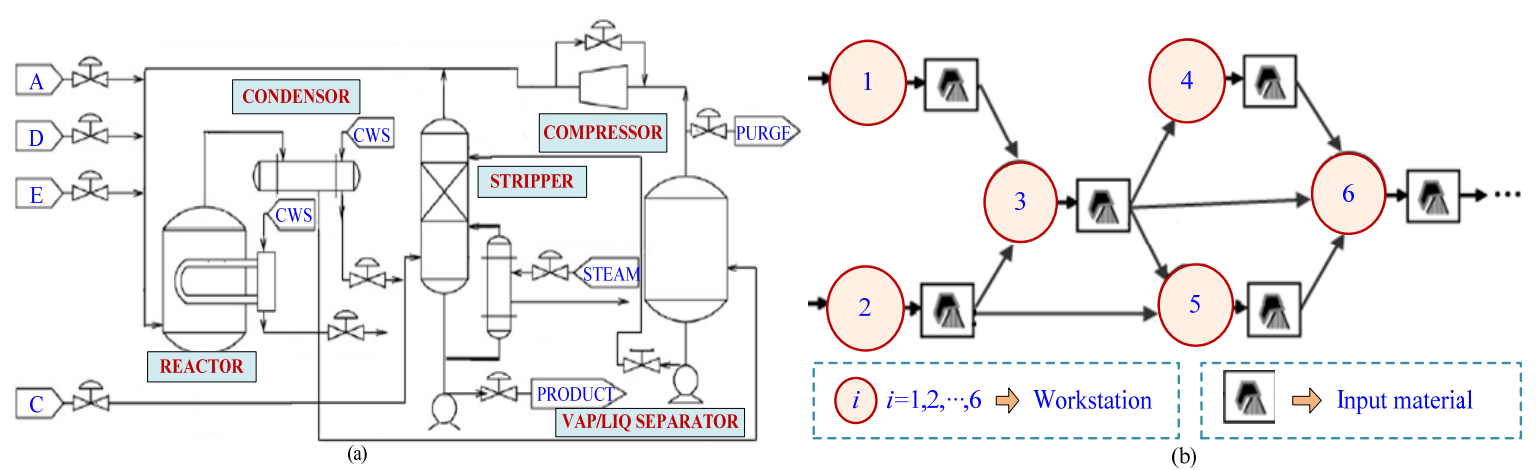

Figure 1. Process configurations:(a) a complex chemical system; (b) an assembly/convergent system

Sufficient sensor deployment is helpful to improve the diagnosis capacity, but inappropriate sensor placement can only increase costs and reduce the system diagnosis efficiency[16].Therefore, appropriate sensor deployment is crucial for an effective diagnosis system design. The general modules of sensor deployment are cause-effect relationship modeling, optimization basis, and optimization algorithm. This paper majorly reviews the above modules influencing the efficiency of sensor measurements on faulty symptoms. And, the challenges and opportunities are discussed with some key conclusions.

\section{Cause-effect Relationship Model}

It is the cornerstone for optimal sensor deployment problems to model the cause-effect relationship between system faults and sensor measurements[17]. The cause-effect model of the sensor-fault is not only the cornerstone but reveals the diagnostic relationship between sensor and fault. Therefore, many complex mathematical models have been constructed by researchers.

Pourali et al.[18] developed a bayesian sensor placement optimization (BSPO) methods that utilized Bayesian networks for modeling, updating, and reasoning the causal relationships and uncertainties to find optimum sensor locations on a system's logic diagram. KiongTan et al.[19] proposed a sensor placement approach by measure and infer the actual vibration spectrum at a critical point, which is associated with one or more sensitive frequencies. Mendibil et al.[20]developed a experimental study by place pressure and temperature sensors in the runner system and the mould micro-featured cavity on micro-injection moulding. Sensor signals is correlated with quality deviations using the confocal microscopy to diagnose the injected micro-parts quality. Oromiehie et al.[21] developed an experimental study on glass fibre/high-density polyethylene laminates with embedded FBG sensors for manufacturing process monitoring. The FBG sensors are used to monitor the reflected wavelengths related to pressure and temperature.

For the purpose of deviation diagnosis in a multistation assembly process, optimal sensor allocation methodologies are usually developed base on a state-space model [16, 22-36]. As shown in Fig. 2, the station-indexed state space model can be represented as

$$
\begin{aligned}
& X_{k}=A_{k-1} X_{k-1}+B_{k} P_{k}+\xi_{k} \\
& Y_{k}=C_{k} X_{k}+\eta_{k}, k \in\{1,2, \cdots, N\}
\end{aligned}
$$

Where $\mathrm{k}$ is the station index, $\mathrm{N}$ the number of stations, $\mathrm{X}_{\mathrm{k}}$ and $\mathrm{Y}_{\mathrm{k}}$ are the product dimensional deviation and the sensor measurements at station $\mathrm{k}$, respectively. $\mathrm{P}_{\mathrm{k}}$ is the random deviations related with fixture locators at station $\mathrm{k}$. A, B and $\mathrm{C}$ are the state matrix. $\xi$ and $\eta$ are un-modeled higher order terms[37]. Wang et al.[38] used statistical analyses of the Fisher information matrix and the prediction matrix for the optimal sensor locations in an automated Coordinate Checking Fixture. The cause-and-effect relation is mathematically described as follows 


$$
\begin{aligned}
& \{\delta d\}_{m \times 1}=\gamma\{\delta \phi\}_{n \times 1} \\
& \gamma=\left(\left[J_{22}\right]-\left[J_{21}\right]\left[J_{11}\right]^{-1}\left[J_{12}\right]\right)_{m \times n}
\end{aligned}
$$

Where $\{\delta \mathrm{d}\} \mathrm{m} \times 1$ corresponds to the position changes measured by the actual $\mathrm{m}$ position sensors. $\gamma$ is the sensitivity matrix related with the deviations in the $\mathrm{n}$ geometric parameters of the part to the changes in the $\mathrm{m}$ sensor readings. $\{\delta \varphi\}_{\mathrm{n} \times 1}$ is the shape deviations.

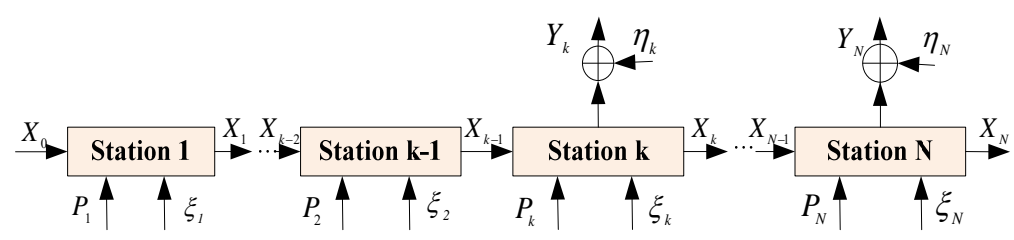

Figure 2. Information flow in multi-station manufacturing

Graph theory, such as Digraph (DG) or signed digraph (SDG) technique, has been applied in the optimal sensor deployment to model the cause-effect relationship between the system faults and sensor measurements by solving a particularly sensor deployment problem to maximize the system reliability[15, 39-44]. A quantitative cause-effect graph (QCEG), illustrated in Fig. 3, have been developed by us to handle the heterogeneity among the properties of sensors and faults to facilitate the monitoring of single-station multistep manufacturing process (SMMP) [17, 45].

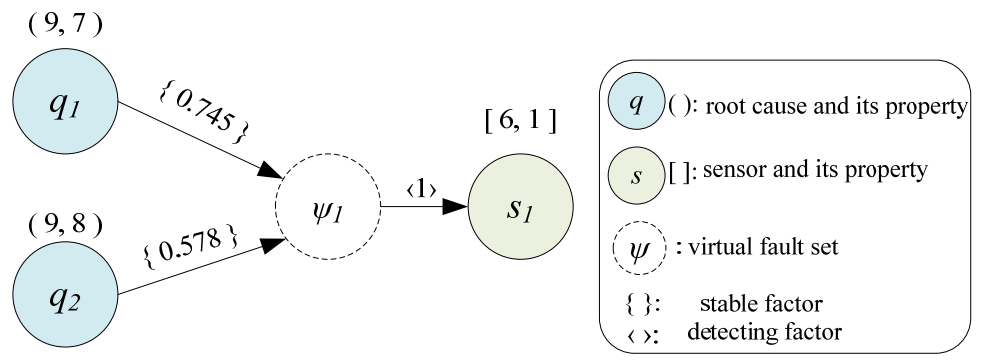

Figure 3. A QCEG for sensor deployment



Figure 4. Inference engine block diagram

A Bayesian Network (BN) is also employed to represent the causal relationships among the physical variables in Distributed Sensor Networks(DSN) to detect the system abnormality[18, 46-49]. The inference engine has three major blocks shown in Fig. 4. Li et al.[50-52] studied the sensor deployment issues related concerns such as coverage, connectivity, and energy efficiency in wireless sensor networks. Fig. 5 shows an example of depicting the coverage of mobile sensor networks where the solid disks being covered at the given time are instant[53]. 


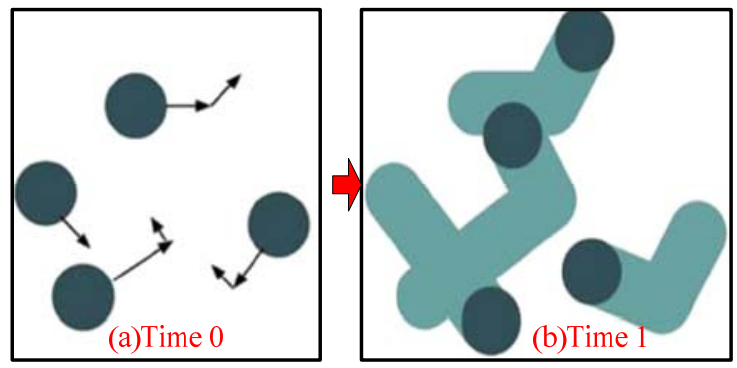

Figure 5. An example of coverage of mobile sensor network

\section{Optimization Basis}

The initial optimization basis for sensor distribution researches are mainly aimed at a single target. A general form can be taken as the following bilevel formulation:

$$
\begin{aligned}
& \min _{x} F(x, y) \\
& \text { s.t. } H(x, y)=0 \\
& G(x, y) \leq 0
\end{aligned}
$$

Where $x, F(x, y), y$ denote decision variables, outer objective function, inner decision variables, respectively. It is usually called a leader-follower problem, where $x$ is decided by the leader while $y$ is decided by the follower[54].

$\mathrm{Lu}$ et al. [55] established the quadrilateral array location equations for an acoustic emission location, which is converted to an optimization problem.

$$
\begin{aligned}
& \min f(x, y)=f_{1}^{2}(x, y)+f_{2}^{2}(x, y) \\
& \text { s.t. } x_{1} \leq x \leq x_{3}, y_{1} \leq y \leq y_{3}
\end{aligned}
$$

Where $f_{1}$ and $f_{2}$ are the ratio of Euclidean distance of acoustic emission propagation. To improve the performance and reduce the computational cost of the robot placement problem, Sheng et al.[56] studied the weighted set-covering problem to integrate kinematics constraint into the vision sensor planning.

$$
\begin{aligned}
& \min \quad \mathrm{z}=\sum_{j=1}^{N_{s}} c_{j} x_{j} \\
& \text { s.t. } \sum_{j=1}^{N_{s}} a_{i j} x_{j} \geq 1, \quad i=1,2, \cdots, N \times M \\
& \quad \text { with } x_{j}=0 \text { or } 1, j=1,2, \cdots, N_{s}
\end{aligned}
$$

Here, $a_{i j}=1$ means cell $i$ belongs to index set $S_{j}$, otherwise not. Variable $x_{j}$ means the index set $S_{j}$ is selected, otherwise not. Bastani et al.[23] proposed an optimal sensor placement based on compressive sensing theory to diagnose the process faults in multi-station assembly processes, which can be formulated as the optimization problem:

$$
\begin{aligned}
& \min \mu_{\text {avg }}(D) \\
& \text { s.t. } \alpha \in F, u \in S
\end{aligned}
$$

Where $\boldsymbol{\alpha}$ refers to the station assignment of the sensors; $u$ denotes the locations of the sensors on the parts, $F$ and $S$ represent the sensor's possible station assignments and feasible locations on the parts, respectively. 
An optimal sensor allocation is investigated to achieve the desired diagnosability with the minimum cost in a multi-station manufacturing system[16, 30, 57]. Chen et al.[25] developed an optimal assignment of locating pin wear rates to maximize the system reliability, which can be formulated as:

$$
\begin{aligned}
& \mu^{*}=\arg \min _{\mu}\left\{s_{K}\right\} \\
& \text { s.t. } C_{p} \leq C_{\max }, \mu_{\min } \leq \mu_{i j}(\Delta) \leq \mu_{\max }, \forall i, j
\end{aligned}
$$

Where $\mu=\left[\mu_{1,1}(\Delta) \mu_{1,1}(\Delta) \ldots \mu_{\mathrm{L}, \mathrm{nL}}(\Delta)\right]^{\mathrm{T}}$ and $\mathrm{C}_{\max }$ is the budget for the total tooling fabrication cost. Tyagi et al.[28, 31] developed an optimal design of fixture layout to minimize the sensitivity $\mathrm{S}$ max while satisfying the geometric and other constraints. The optimization problem can be expressed as follows

$$
\begin{aligned}
& \min _{\psi(\ell)} \mathrm{S}_{\max }(\psi(\ell))=\lambda_{\max }\left(\mathrm{M}^{\mathrm{T}} \mathrm{M}\right) \\
& \text { s.t. } \mathrm{C}(\psi(\ell)) \geq 0
\end{aligned}
$$

Where $\psi(\ell)$ denotes the locators position, $\mathrm{C}(\cdot)$ is the geometrical constraint on the principal locating points. Li et al.[47] translated the optimal sensor allocation problem into a set-covering problem to minimize the total sensing cost as well as satisfy a prescribed detectability requirement.

$$
\begin{aligned}
& \min W=\sum_{j=1}^{q} w\left(\Omega_{j}\right) \\
& \text { s.t. } \bigcup_{j=1}^{q} C^{\left\{\Omega_{j}\right\}}=X
\end{aligned}
$$

Where $\mathrm{w}\left(\Omega_{\mathrm{j}}\right)$ is the cost of sensor $\Omega_{\mathrm{j}} . \mathrm{C}^{\{\Omega \mathrm{j}\}}$ is the union of the duty sets.

With the advent of distributed sensing networks (DSNs), it is necessary to further study the multitarget-based sensor layout strategy. An optimization formulation aimed at maximizing the reliability of the fault monitoring system is proposed [15, 39, 40, 42, 44]. Benatia et al.[58] proposed a integrated multi-objectives deployment strategy by employing genetic algorithms under coverage, cost, connectivity constraints, to get near optimal solution for WSN deployment. We also developed a multi-objective optimization, which minimizes the fault unobservability, maximizes the system stability, and minimizes the cost for the whole system, under the constraints on detectability, stationarity, and limited resources[17, 45]. Mathematically, this can be expressed as:

$$
\begin{aligned}
& R_{s}=\min _{j}\left\{\max _{i}\left[\log \left(P_{f}(j)\right)+x_{i} \tau_{i j} \log \left(P_{s}(i)\right)\right]\right\} \\
& D_{s}=\max _{j}\left[\min _{i}\left(x_{i} \varphi_{j i}\right)\right] \\
& \min : C=\sum_{i} c_{i} x_{i} \\
& \text { s.t. } \prod_{j \in Q}\left(\sum_{i \in S} \tau_{j i} x_{i}\right)>0, \frac{1}{J} \sum_{j}\left[\max _{i \in S}\left(x_{i} \varphi_{j i}\right)\right] \leq U_{s}
\end{aligned}
$$

where $P_{f}(i)$ is fault occurrence probability for fault $i, P_{s}(i)$ is sensor failure probability for sensor $i$, and $x_{i}$ is the number of sensor $i$.

\section{Optimization Algorithm}

After establishing the optimization basis, a variety of optimization algorithms, from mathematic programming to heuristic search[28, 59], have been employed to optimize the sensor deployment. 
Based on the effective independence(EfI) method[60], Sheng et al.[61] proposed a sensor-placement strategy to monitor a rolling element bearing with a localized structural defects in the inner or outer raceway. The results show that EfI-based optimal sensor locations are significantly better in identifying the outer raceway defect than nonoptimal locations (from $40 \%$ to over $80 \%$ ). Ghani et al.[62-64] proposed a finite element method (FEM) simulation methodology for the optimum sensor Location. Li et al.[65] proposed a fuzzy clustering algorithm to optimize sensor arrangement for field-programmable gate array temperature measurements. A BSPO algorithms is developed by Pourali et al.[18] for sensor placement optimization under uncertainty. The details of BSPO steps are shown in Fig. 6. Chen et al.[25] develop a Quality and Reliability chain (QR chain) model to integrate the process and product design information of assembly systems.

A state space model is employed to study the variation propagation, and the optimal solution is derived based on the analytical form of system reliability[66]. Wang et al.[38] proposed a statistical analyses of the Fisher information matrix and the prediction matrix using the Powell's direct search to obtain an optimal sensor locations for an automated coordinate checking fixture. Liu et al.[27, 67, 68] developed an Exchange algorithms for sensor optimal layout or fixture layout design for the diagnosis of dimensional variation sources in assembly processes. Ding et al.[16] [37] develop a backward-propagation strategy for optimal allocation of sensors to determine the sensing station and number of sensors in a multi-station assembly process. Khan et al.[69-71] proposed a gradient-based search to achieve an optimal sensor distribution for the diagnosability in a multi-fixture assembly of sheet metal parts.



Figure 6. BSPO flow chart

For the maximum-reliability optimization problem, a greedy search heuristic has been developed $[15,39-41,72]$. Wu et al.[17, 44] investigated a multiple-objective optimization involved in the sensor deployment. They developed a lexicographical mixed integer linear programming and greedy search for sensor deployment optimization. As a heuristic algorithm, greedy search tend to be more effective in finding good feasible solutions as a new approach for a posteriori articulation of preferences[73].

By employing the principles of GAs, many optimal sensor placement are developed in a complex system to optimize several competing evaluation criteria[26, 33, 58, 74-79]. Ren et al.[31] developed a data-mining guided GA to solve the sensor distribution problem to achieve a maximal variance detection capability in a multi-station assembly process. The data-mining-guided GA demonstrates an improvement compared to the existing alternatives. According to the acoustic emission signals 
picked up by four FBG sensors, Lu et al.[55] proposed an improved PSO algorithm to achieve the acoustic emission optimal location. By using the information fusion of multiple standards PSO[80], the process of the improved PSO is realized, as shown in Fig. 7. In Fig. 7, PSO $i, i=1,2,3, \cdots, 10$ denotes the $i$-th standard PSO, ZY $i, i=1,2,3, \cdots, 10$ represents the optimal value of the $i$-th standard PSO. Liu et al.[48] developed a Best Allocation Subsets by Intelligent Search (BASIS) to obtain the optimal sensor allocation at minimum cost under different specified Average Run Length (ARL) requirements in a manufacturing system. In addition, highly optimized tolerance (HOT) algorithms[28], simulated annealing[30,81], and shuffled frog leaping algorithm[45],ect., are also proposed to deal with the multi-objective sensor optimization distribution problem.

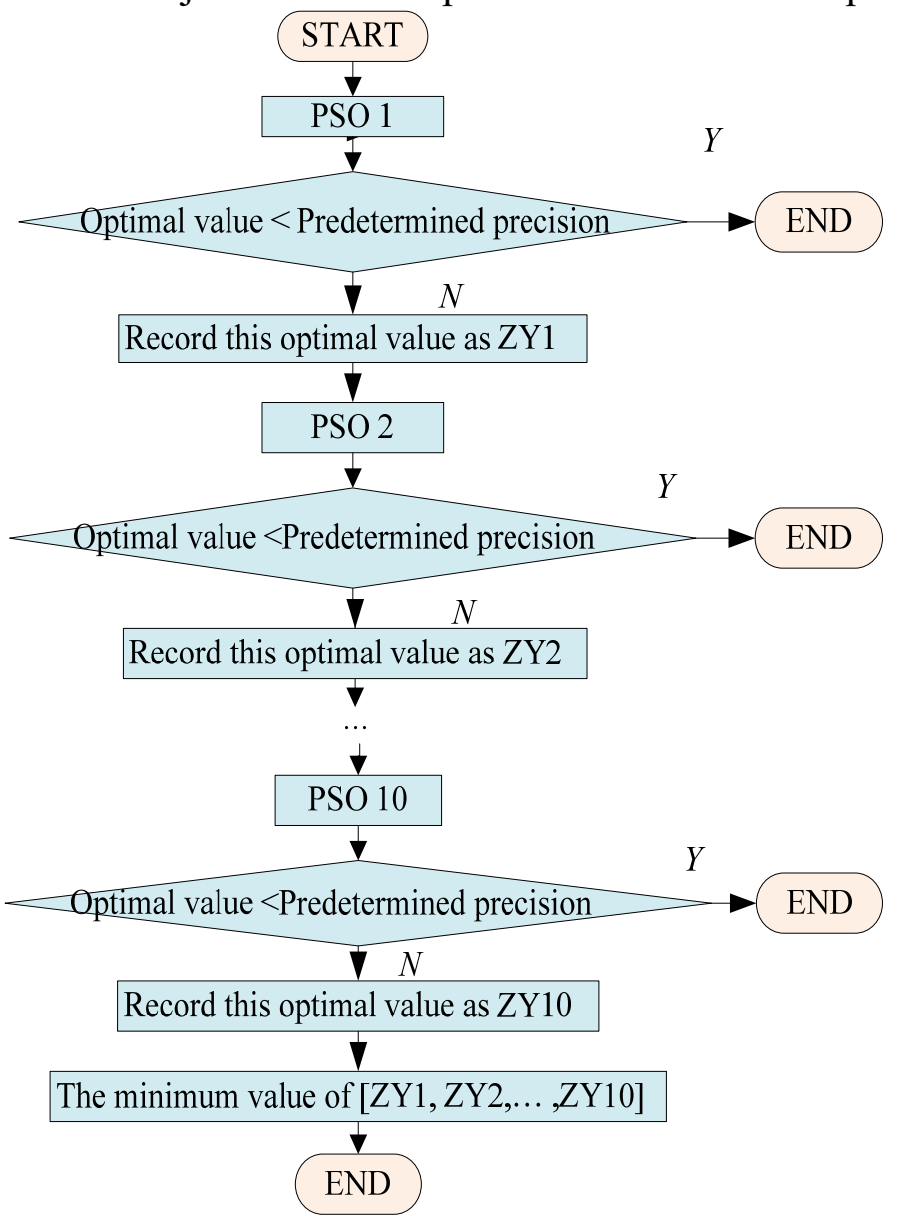

Figure 7. The process of the improved PSO

\section{Challenges for Future Research}

(1) The fault diagnosis ability can be improved by optimizing the sensor layout in discrete-part manufacturing system. However, sensor distribution strategies usually focus on different aspects of a practical problem. The physically meaningful variables and parameters employed in sensor distribution strategy are limited by the insight from its solution. Therefore, a high level operational strategy cannot be constituted in a complicated discrete-part manufacturing system.

(2) Sensor deployment strategies are usually predetermined in the most current literatures. However, the discrete-part manufacturing system is a dynamic system that state changes with time. an adaptive strategy for sensor deployment seems to be more realistic and required in the further.

(3) The heterogeneous sensor systems have been modeled and characterized in some literatures. However, no systematic approach towards optimal sensor deployment strategy for such a heterogeneous system has yet been reported especially for sensor deployment strategies explicitly designed for DSN in a multi-station process. 
(4) Many kinds of heuristic methods are employed in the current sensor deployment strategy; However, their convergence speed is relatively slow and the solution may not be the optimal one. Therefore, the simplified algorithms based on the theoretical analysis needs to be further explored.

\section{Conclusion}

(1) Many complex optimal strategies have been constructed to model cause-effect relationship between system faults and sensor measurements, which including Bayesian networks, experimental mothed, the effective independence method, etc. For the purpose of deviation diagnosis in a multi-station assembly process, optimal sensor allocation methodologies are usually developed base on a state-space model. Moreover, graph theory, such as the Digraph, signed digraph, Fuzzy bipartite graph, and Quantitative cause-effect graph, has been applied to avoid using cumbersome mathematical terms to model the cause-effect relationship between the system faults and sensor measurements in the optimal sensor deployment.

(2) For single-objective optimal, more studies define the optimization objective as the cost of sensor system. In a multi-station manufacturing system, the optimal sensor allocation is to achieve the desired diagnosability, the system reliability, and the sensitivity, etc. For the multi-objective layout strategy, the optimization objective usually involves the fault unobservability, the system stability, the coverage, and the sensing cost, etc.

(3) Most of the optimization approaches employed for sensor arrangement in discrete-part manufacturing system are the effective independence method, finite element method, fuzzy clustering algorithm, gradient-based search algorithms, exchange algorithms, lexicographical mixed integer linear programming. In addition, for the multi-objective sensor optimization distribution problem, the intelligent optimization algorithms, such as PSO algorithm, genetic algorithm, and shuffled frog leaping algorithm, are also employed in the current literatures.

\section{Acknowledgments}

This work was supported in part by the National Natural Science Foundation of China under Grant No.51075070, in part by the Natural Science Foundation of Anhui Province under Grant No.1708085ME104 and 1808085ME127, in part by the Key Project of Natural Science Foundation of Universities of Anhui Province under Grant No. KJ2017A439 and KJ2016A803. and in part by the Suzhou Univ. Professor (PhD) Scientific Research Foundation under Grant No.2016JB09.

\section{References}

[1]. W. Qiao, and D. Lu, A survey on wind turbine condition monitoring and fault diagnosis - Part I: Components and subsystems, Ieee. T. Ind. Electron.,62 (2015) 6536-6545.

[2]. M. Riera-Guasp, J. A. Antonino-Daviu, and G.-A. Capolino, Advances in electrical machine, power electronic, and drive condition monitoring and fault detection: state of the art, Ieee. T. Ind. Electron.,62 (2015) 1746-1759.

[3]. Y. Lei, J. Lin, Z. He et al., A review on empirical mode decomposition in fault diagnosis of rotating machinery, Mech. Syst. Signal Pr.,35 (2013) 108-126.

[4]. D. K. Pradhan, Fault-tolerant computer system design, Prentice-Hall, Upper Saddle River, NJ 1996.

[5]. R. J. Patton, P. M. Frank, and R. N. Clark, Issues of fault diagnosis for dynamic systems, Springer Science \& Business Media, London, UK, 2013.

[6]. A. Siddhpura, and R. Paurobally, A review of flank wear prediction methods for tool condition monitoring in a turning process, Int. J. Adv. Manuf. Tech,65 (2013) 371-393. 
[7]. A. J. Torabi, M. J. Er, X. Li et al., Application of clustering methods for online tool condition monitoring and fault diagnosis in high-speed milling processes, IEEE Syst. J.,10 (2016) 721-732.

[8]. Y. Lei, D. Han, J. Lin et al., Planetary gearbox fault diagnosis using an adaptive stochastic resonance method, Mech. Syst. Signal Pr.,38 (2013) 113-124.

[9]. Y. Lei, J. Lin, M. J. Zuo et al., Condition monitoring and fault diagnosis of planetary gearboxes: A review, Measurement,48 (2014) 292-305.

[10]. K. Bastani, Z. Kong, W. Huang et al., Fault diagnosis using an enhanced relevance vector machine (RVM) for partially diagnosable multistation assembly processes, IEEE T. Auto. Sci. Eng., 10 (2013) 124-136.

[11]. Y. Liu, X. Ye, and S. Jin, A bayesian based process monitoring and fixture fault diagnosis approach in the auto body assembly process, J. Shanghai Jiaotong Univ. (Sci.),21 (2016) 164-172.

[12]. H. Shui, X. Jin, and J. Ni, An Anomaly Detection and Diagnosis Method Based on Real-Time Health Monitoring for Progressive Stamping Processes. J. Dermatol. Sci., 16 (2015) 170-177.

[13]. G. Huang, 10 emerging technologies that will change your world, Technol. Rev.,107 (2004) $32-50$.

[14]. S. S. Mandroli, A. K. Shrivastava, and Y. Ding, A survey of inspection strategy and sensor distribution studies in discrete-part manufacturing processes, Iie Trans.,38 (2006) 309-328.

[15]. M. Bhushan, and R. Rengaswamy, Comprehensive design of a sensor network for chemical plants based on various diagnosability and reliability criteria. 2. Applications, Ind. Eng. Chem. Res.,41 (2002) 1840-1860.

[16]. Y. Ding, P. Kim, D. Ceglarek et al., Optimal sensor distribution for variation diagnosis in multistation assembly processes, Ieee. T. Robotic. Autom, 19 (2003) 543-556.

[17]. Z. Wu, S.-J. Hsieh, and J. Li, Sensor deployment based on fuzzy graph considering heterogeneity and multiple-objectives to diagnose manufacturing system, Robot. Cim-int. Manuf.,29 (2013) 192-208.

[18]. M. Pourali, and A. Mosleh, A Bayesian approach to sensor placement optimization and system reliability monitoring, P.I. Mech. Eng.O-J. Ris.,227 (2013) 327-347.

[19]. P. V. E. C. S. K. KiongTan, Approach towards sensor placement, selection and fusion for real-time condition monitoring of precision machines, Mech. Syst. Signal Pr.,68-69 (2016) $105-124$.

[20]. X. Mendibil, I. Llanos, H. Urreta et al., In process quality control on micro-injection moulding: the role of sensor location, Int. J. Adv. Manuf. Tech,89 (2017) 3429-3438.

[21]. E. Oromiehie, B. G. Prusty, P. Compston et al., In situ process monitoring for automated fibre placement using fibre Bragg grating sensors, Struct. Heath Monit.,15 (2016) 706-714.

[22]. D.-M. Chang, C.-C. Yu, and I.-L. Chien, Arrangement of multi-sensor for spatio-temporal systems: application to sheet-forming processes, Chem. Eng. Sci.,56 (2001) 5709-5717.

[23]. K. Bastani, Z. Kong, W. Huang et al., Compressive sensing-based optimal sensor placement and fault diagnosis for multi-station assembly processes, Iie Trans., 48 (2016) 462-474.

[24]. Y. Ding, and D. Apley, Guidelines for placing additional sensors to improve variation diagnosis in assembly processes, Int. J. Prod. Res.,45 (2007) 5485-5507.

[25]. Y. Chen, J. Jin, and J. Shi, Integration of dimensional quality and locator reliability in design and evaluation of multi-station body-in-white assembly processes, Iie Trans.,36 (2004) 827-839. 
[26]. N. Shukla, D. Ceglarek, and M. K. Tiwari, Key characteristics-based sensor distribution in multi-station assembly processes, J. Intell. Manuf.,26 (2015) 43-58.

[27]. C. Q. Liu, Y. Ding, and Y. Chen, Optimal coordinate sensor placements for estimating mean and variance components of variation sources, Iie Trans.,37 (2005) 877-889.

[28]. S. Tyagi, N. Shukla, and S. Kulkarni, Optimal design of fixture layout in a multi-station assembly using highly optimized tolerance inspired heuristic, Appl. Math. Model.,40 (2016) 6134-6147.

[29]. H. R. Shaker, and M. Tahavori, Optimal sensor and actuator location for unstable systems, J. Vib. Control,19 (2013) 1915-1920.

[30]. N. Shukla, M. Tiwari, and R. Shankar, Optimal sensor distribution for multi-station assembly process using chaos-embedded fast-simulated annealing, Int. J. Prod. Res.,47 (2009) 187-211.

[31]. Y. Ren, and Y. Ding, Optimal sensor distribution in multi-station assembly processes for maximal variance detection capability, Iie Trans.,41 (2009) 804-818.

[32]. H. OLOOMI, and M. E. SAWAN, Optimal sensor placement in decentralized control systems, Int. J. Syst. Sci.,20 (1989) 939-943.

[33]. I. Bruant, L. Gallimard, and S. Nikoukar, Optimization of piezoelectric sensors location and number using a genetic algorithm, Mech. Adv. Matl. Struct.,18 (2011) 469-475.

[34]. J. V. Abellan-Nebot, J. Liu, and F. R. Subirón, Quality prediction and compensation in multi-station machining processes using sensor-based fixtures, Robot. Cim-int. Manuf.,28 (2012) 208-219.

[35]. J. Jin, and J. Shi, State space modeling of sheet metal assembly for dimensional control, J. Manuf. Sci. E-T ASME,121 (1999) 756-762.

[36]. O. Tonomura, S. Nagahara, J.-i. Kano et al., Sensor location for effective fault diagnosis in micro chemical processes, IFAC Proceedings Volumes, 42 (2009) 309-314.

[37]. L. Xinmin, T. Zhaoqing, and L. Zhongqin, A simplified method for optimal sensor distribution for process fault diagnosis in multistation assembly processes, J. Manuf. Sci.E-T ASME,130 (2008) 051002.

[38]. Y. Wang, and S. R. Nagarkar, Locator and sensor placement for automated coordinate checking fixtures, J. Manuf. Sci. E-T ASME,121 (1999) 709-719.

[39]. M. Bhushan, and R. Rengaswamy, Comprehensive design of a sensor network for chemical plants based on various diagnosability and reliability criteria. 1. Framework, Ind. Eng. Chem. Res.,41 (2002) 1826-1839.

[40]. M. Bhushan, and R. Rengaswamy, Design of sensor location based on various fault diagnostic observability and reliability criteria, Comput. Chem. Eng.,24 (2000) 735-741.

[41]. R. Raghuraj, M. Bhushan, and R. Rengaswamy, locating sensors in complex chemical plants based on fault diagnostic observability criteria, Aiche J.,45 (1999) 310-322.

[42]. F. Yang, D. Xiao, and S. L. Shah, Optimal sensor location design for reliable fault detection in presence of false alarms, Sensors,9 (2009) 8579-8592.

[43]. H. Qi, S. S. Iyengar, and K. Chakrabarty, Distributed sensor networks - a review of recent research, J. Franklin I.,338 (2001) 655-668.

[44]. M. Bhushan, S. Narasimhan, and R. Rengaswamy, Robust sensor network design for fault diagnosis, Comput. Chem. Eng.,32 (2008) 1067-1084. 
[45]. K. He, M. Jia, and Q. Xu, Optimal sensor deployment for manufacturing process monitoring based on quantitative cause-effect graph, IEEE T. Autom. Sci. Eng.,13 (2016) 963-975.

[46]. M. Askarian, G. Escudero, M. Graells et al., Fault diagnosis of chemical processes with incomplete observations: A comparative study, Comput. Chem. Eng.,84 (2016) 104-116.

[47]. J. Li, and J. Jin, Optimal sensor allocation by integrating causal models and set-covering algorithms, Iie Trans.,42 (2010) 564-576.

[48]. K. Liu, and J. Shi, Objective-oriented optimal sensor allocation strategy for process monitoring and diagnosis by multivariate analysis in a Bayesian network, Iie Trans.,45 (2013) 630-643.

[49]. M. S. Sayed, and N. Lohse, Ontology-driven generation of Bayesian diagnostic models for assembly systems, Int. J. Adv. Manuf. Tech,74 (2014) 1033-1052.

[50]. J. Li, L. L. Andrew, C. H. Foh et al., Connectivity, coverage and placement in wireless sensor networks, Sensors,9 (2009) 7664-7693.

[51]. E. N. Gilbert, Random plane networks, J. Am. Soc. Mass. Spect.,9 (1961) 533-543.

[52]. S. Abdollahzadeh, and N. J. Navimipour, Deployment strategies in the wireless sensor network: a comprehensive review, Comput. Commun.,91 (2016) 1-16.

[53]. B. Liu, O. Dousse, P. Nain et al., Dynamic coverage of mobile sensor networks, Ieee. T. Parall. Distr.,24 (2013) 301-311.

[54]. A. Kumar, M. Baldea, and T. F. Edgar, On optimal sensing and actuation design for an industrial scale steam methane reformer furnace, Aiche J.,62 (2016) 3225-3237.

[55]. S. Lu, M. Jiang, Q. Sui et al., Acoustic emission location on aluminum alloy structure by using FBG sensors and PSO method, J. Mod. Optic.,63 (2016) 742-749.

[56]. W. Sheng, N. Xi, M. Song et al., CAD-guided sensor planning for dimensional inspection in automotive manufacturing, IEEE/ASME T.Mech.,8 (2003) 372-380.

[57]. J.-w. Sun, L.-f. Xi, E.-s. Pan et al., Design for diagnosability of multistation manufacturing systems based on sensor allocation optimization, Comput. Ind.,60 (2009) 501-509.

[58]. M. A. Benatia, M. h. Sahnoun, D. Baudry et al., Multi-Objective WSN Deployment Using Genetic Algorithms Under Cost, Coverage, and Connectivity Constraints, Wireless Pers.1 Commun. ,94 (2017) 2739-2768.

[59]. G. Onwubolu, and M. Clerc, Optimal path for automated drilling operations by a new heuristic approach using particle swarm optimization, Int. J. Prod. Res.,42 (2004) 473-491.

[60]. D. C. Kammer, "Sensor placement for on-orbit modal identification and correlation of large space structures." in American Control Conference, San Diego, CA, USA, pp. 2984-2990.

[61]. S. Sheng, L. Zhang, and R. X. Gao, A systematic sensor-placement strategy for enhanced defect detection in rolling bearings, IEEE Sensors Journal,6 (2006) 1346-1354.

[62]. J. A. Ghani, P. S. Jye, C. H. C. Haron et al., Determination of sensor location for cutting tool deflection using finite element method simulation, P.I. Mech. Eng.C-J. MEC.,226 (2012) 2373-2377.

[63]. F. Bachmann, A. E. Bergamini, and P. Ermanni, Optimum piezoelectric patch positioning: A strain energy-based finite element approach, J. Intel. Mat. Syst. Str.,23 (2012) 1575-1591.

[64]. A. Belloli, and P. Ermanni, Optimum placement of piezoelectric ceramic modules for vibration suppression of highly constrained structures, Smart Mater. Struc.,16 (2007) 1662. 
[65]. J. Li, S. Feng, Y. Zhang et al., Optimized thermal sensor allocation for field-programmable gate array temperature measurements based on self-heating test, Microelectr. J.,60 (2017) 55-59.

[66]. Y. Ren, Y. Ding, and F. Liang, Adaptive evolutionary Monte Carlo algorithm for optimization with applications to sensor placement problems, Stat. Comput.,18 (2008) 375-390.

[67]. J. A. Camelio, S. J. Hu, and H. Yim, Sensor Placement for Effective Diagnosis of Multiple Faults in Fixturing of Compliant Parts. J Manuf. Sci. E-T ASME,127(2003) 373-380.

[68]. P. Kim, and Y. Ding, Optimal design of fixture layout in multistation assembly processes, IEEE T. Autom. Sci. Eng., 1 (2004) 133-145.

[69]. A. Khan, D. Ceglarek, and J. Ni, Sensor location optimization for fault diagnosis in multi-fixture assembly systems, J. Manuf. Sci. E-T ASME,120 (1998) 781-792.

[70]. A. Khan, D. Ceglarek, J. Shi et al., Sensor optimization for fault diagnosis in single fixture systems: a methodology, J. Manuf. Sci. E-T ASME,121 (1999) 109-120.

[71]. A. Khan, and D. Ceglarek, Sensor optimization for fault diagnosis in multi-fixture assembly systems with distributed sensing, J. Manuf. Sci. E-T ASME, 122 (2000) 215-226.

[72]. S. Costiner, H. A. Winston, M. R. Gurvich et al., A probabilistic hybrid sensor fusion and optimization approach for aircraft composite components, J. Intel. Mat. Syst. Str.,24 (2013) 2110-2134.

[73]. R. T. Marler, and J. S. Arora, Survey of multi-objective optimization methods for engineering, Struct. Multidiscip. O,26 (2004) 369-395.

[74]. N. S. Sen S, Deb K., Sensor network design of linear processes using genetic algorithms, Comput. Chem. Eng.,22 (1998) 385-390.

[75]. H. Chen, J. A. Clark, J. E. Tapiador et al., Computational Intelligence in Security for Information Systems, Springer, New York ,2009.

[76]. K. Worden, and A. Burrows, Optimal sensor placement for fault detection, Eng. Struct.,23 (2001) 885-901.

[77]. X. Wang, S. Wang, and A. Jiang, "Optimized deployment strategy of mobile agents in wireless sensor networks." in International Conference on Intelligent Systems Design and Applications, IEEE Computer Society, Washington, D.C., pp. 893-898.

[78]. C. C. Yang, and F. W. Ciarallo, Optimized sensor placement for active visual inspection, J. Robotic. Syst., 18 (2001) 1-15.

[79]. B. Jung, J. Cho, and W. Jeong, Sensor placement optimization for structural modal identification of flexible structures using genetic algorithm, J. Mech.1 Sci.Technol.,29 (2015) 2775-2783.

[80]. C. Mendis, S. M. Guru, S. Halgamuge et al., "Optimized sink node path using particle swarm optimization." in International Conference on Advanced Information NETWORKING and Applications, IEEE Computer Society, Washington, D.C., pp. 388-394.

[81]. J. Guo, and H. Jafarkhani, Sensor deployment with limited communication range in homogeneous and heterogeneous wireless sensor networks, IEEE T. Wirel. Commun.,15 (2016) 6771-6784. 